\title{
Erratum
}

Monatsschr Kinderheilkd 2014 · 162:153-155

DOI 10.1007/s00112-013-3065-6

Online publiziert: 6. Februar 2014

(c) Springer-Verlag Berlin Heidelberg 2014

G. Gramer · G.F. Hoffmann · S. Kölker

Sektion für Angeborene

Stoffwechselerkrankungen,

Zentrum für Kinder- und Jugendmedizin,

Universitätsklinikum Heidelberg

\section{Erratum zu: Metabolische Notfalltherapie}

Leider ist es in diesem Beitrag aufgrund eines Schreibfehlers zu fehlerhaften Angaben gekommen. In Tab. 3 „Besonderheiten in der Präanalytik“" heißt es zu Ammoniak:

„Cave! Unterschiedliche Einheiten: $\mu \mathrm{mol} / \mathrm{l}=\mathrm{mg} / \mathrm{dl} \times 0,59^{\text {“ }}$

Korrekt müsste es lauten:

, $\mu \mathrm{mol} / \mathrm{l}=\mu \mathrm{g} / \mathrm{dl} \times 0,59$ “

Wir bitten diesen Fehler zu entschuldigen und künftig die korrigierten Angaben zu beachten.

\section{Korrespondenzadresse}

Dr. G. Gramer

Sektion für Angeborene

Stoffwechselerkrankungen

Zentrum für Kinder- und Jugendmedizin

Universitätsklinikum Heidelberg

Im Neuenheimer Feld 430, 69120 Heidelberg

gwendolyn.gramer@med.uni-heidelberg.de

Die Online-Version des Originalartikels

können Sie unter http://dx.doi.org/10.1007/

s00112-013-2939-y finden. 